\title{
Investigating Jeffery-Hamel flow with high magnetic field and nanoparticle by HPM and AGM
}

Research Article

\author{
A. K. Rostami ${ }^{1}$ M. R. Akbari $^{2}$, D. D. Ganji ${ }^{1 *}$, S. Heydari ${ }^{1}$ \\ 1 Department of Mechanical Engineering, Babol University of Technology, Babol, Iran \\ 2 Department of Civil Engineering, The university of Pardisan Mazandaran and chemical engineering University of Tehran, Iran
}

Received 27 April 2014; accepted 09 July 2014

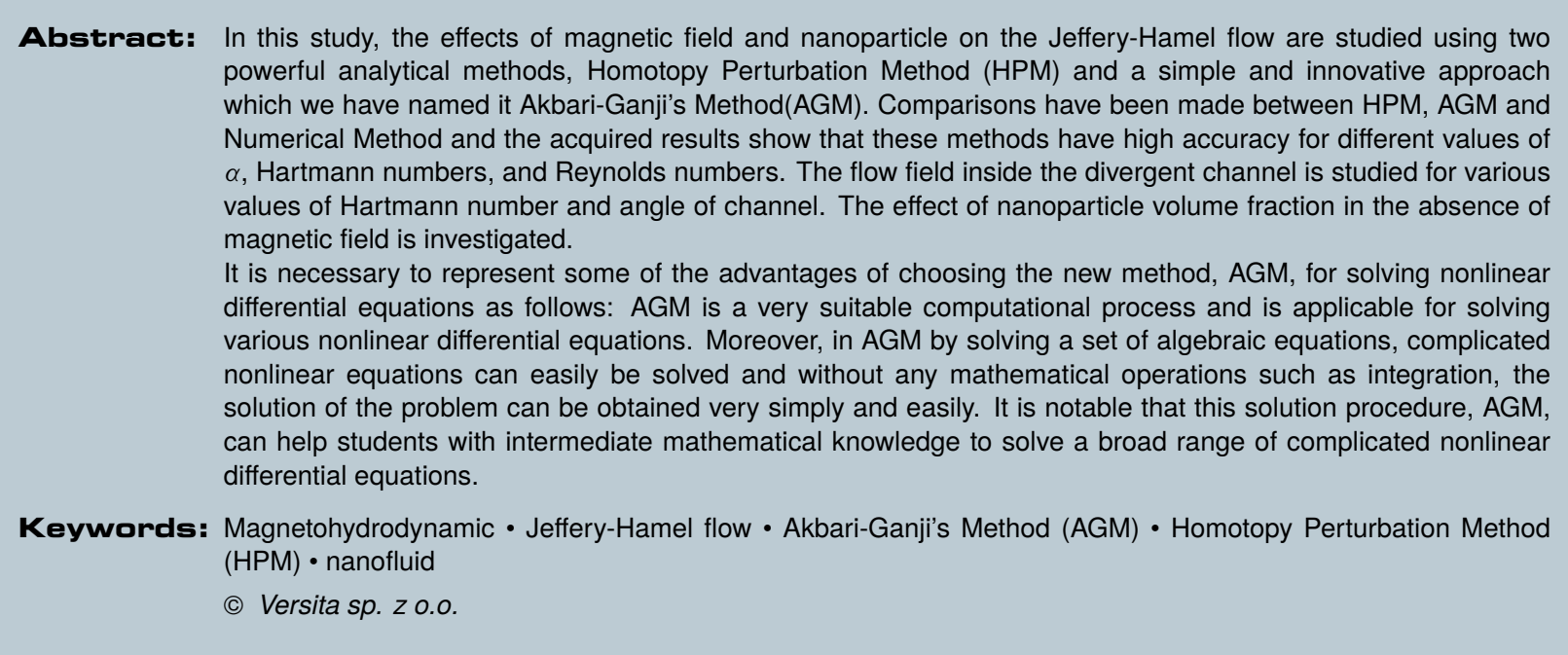

\section{Introduction}

After introducing the problem of the fluid flow through a divergent channel by Jeffery [1] and Hamel [2] in 1915 and 1916, respectively, it is called Jeffery-Hamel flow. On the other hand, the term of magnetohydrodynamic (MHD) was first introduced by Alfven [3] in 1970. The theory

*E-mail: ddg_davood@yahoo.com of MHD is inducing current in a moving conductive fluid in the presence of magnetic field; such induced current results force on ions of the conductive fluid. The study of MHD channel has been a subject of great interest due to its wide applications in designing cooling systems with liquid metals, MHD generators, accelerators, pumps, and flow meters $[4,5]$.

The small disturbance stability of MHD stability of plane-Poiseuille flow was investigated by Makinde and Motsa [6] and Makinde [7] for generalized plane Couette flow. Their results showed that magnetic field had 
stabilizing effects on the flow. Considerable efforts have been done to study the MHD theory for technological application of fluid pumping system in which electrical energy forces the working conductive fluid. Damping and controlling of electrically conducting fluid can be achieved by means of an electromagnetic body force (Lorentz force), which produced by interaction of an applied magnetic field and an electric current that usually is externally supplied. Anwari et al. [8] studied the fundamental characteristics of linear Faraday MHD theoretically and numerically, for various loading configurations. Homsy et al. [9] emphasized on the idea that in such problems, the moving ions drag the bulk fluid with themselves, and such MHD system induces continued pumping of conductive fluid without any moving part. Taking into account the rising demands of modern technology, including chemical production, power station, and microelectronics, there is a need to develop new types of fluids that will be more effective in terms of heat exchange performance. The term 'nanofluid' was envisioned to describe a fluid in which nanometer-sized particles were suspended in conventional heat transfer basic fluids [10]. In fluid mechanics, most of problems are non-linear. It is very important to develop efficient methods to solve them. In this paper, we use two methods to solve the nonlinear differential equation governing on the mentioned problem. One of the used methods in this case study is Homotopy Perturbation Method (HPM) and the other one is a simple and innovative approach which has been called AkbariGanji's Method (AGM).

HPM is an effective and convenient method for both linear and nonlinear equations. This method does not depend on a small parameter. This method was applied for many nonlinear problems some of which are introduced in [1114]. Roozi et al. [15] used HPM to solve nonlinear parabolic-hyperbolic partial differential equations and presented examples of one-dimension and two-dimensions to show the ability of the method for such equations. Ganji and Sadighi [16] applied HPM for solving non-linear heat transfer and porous media equations also they introduced HPM to obtain the exact solutions of linear and nonlinear Schrodinger equations [17]. Sadighi and Ganji [18] obtained the exact solutions of the Laplace equation with Dirichlet and Neumann boundary conditions using HPM. Ziabakhsh and Domairry [19] have studied the natural convection of a non-Newtonian fluid between two infinite parallel vertical flat plates and the effects of the non-Newtonian nature of fluid on the heat transfer by HPM. Ganji et al. [20] considered two known nonlinear systems which are different in phenomena but same in practice. The resulted nonlinear differential equation was separately solved by using the HPM and compared with numerical solution. Also Khavaji et al. [21] applied HPM for finding the large deflections subject in compliant mechanisms.

The other used method in this paper is AGM, which is a new powerful method for solving nonlinear differential equations especially in the field of fluid mechanics. It is beneficial to represent some of the advantages of choosing this approach for solving nonlinear differential equations as follows: AGM is a very suitable computational process and is usable for solving various nonlinear differential equations. Moreover, in AGM by solving a set of algebraic equations, complicated nonlinear equations can easily be solved and without any mathematical operations such as integration, the solution of the problem can be obtained very simply and easily. It is notable that this solution procedure, AGM, can help students with intermediate mathematical knowledge to solve a broad range of complicated nonlinear differential equations.

It is worthwhile to note that in AGM considering three or four sentences of the series of polynomials which are considered as the answer of differential equations is sufficient to obtain reliable solutions, and always by increasing the number of sentences the solution gets closer to the exact solution while in other methods the error might increase or decrease. For example, in this problem by adding a sentence to the series of polynomials the solutions get much more accurate.

\section{Problem description and governing equations}

Consider a system of cylindrical polar coordinates $(r, \theta, z)$ in which steady two-dimensional flow of an incompressible conducting viscous fluid from a source or sink at channel walls lie in planes, and intersect in the axis of $z$. Assume purely radial motion which means that there is no change in the flow parameter along the $z$-direction. The flow depends on $r$ and $\theta$, and further we assume that there is no magnetic field in the $z$-direction. The reduced forms of continuity, Navier-Stokes, and Maxwell's equations [22] are:

$$
\begin{gathered}
\frac{\rho_{n f}}{r} \frac{\partial(r u(r, \theta))}{\partial r}(r u(r, \theta))=0 \\
u(r, \theta) \frac{\partial u(r, \theta)}{\partial r}=-\frac{1}{\rho_{n f}} \frac{\partial P}{\partial r}+v_{n f}\left(\frac{\partial^{2} u(r, \theta)}{\partial r^{2}}+\right. \\
\left.\frac{1}{r} \frac{\partial u(r, \theta)}{\partial r}+\frac{1}{r^{2}} \frac{\partial^{2} u(r, \theta)}{\partial \theta^{2}}-\frac{u(r, \theta)}{r^{3}}\right)-\frac{\sigma B_{0}^{2}}{\rho_{n f} r^{2}} u(r, \theta) \\
\frac{1}{\rho_{n f}} \frac{\partial P}{\partial \theta}-\frac{2 v_{n f}}{r^{2}} \frac{\partial u(r, \theta)}{\partial \theta}=0
\end{gathered}
$$


where $B_{0}$ is the electromagnetic induction, $\sigma$ is the conductivity of the fluid, $u(r)$ is the velocity along radial direction, $P$ is the fluid pressure, $v_{n f}$ is the coefficient of kinematic viscosity, and $\rho_{n f}$ is the fluid density. The effective density $\rho_{n f}$, the effective dynamic viscosity $\mu_{n f}$, and the kinematic viscosity of $v_{n f}$ the nanofluid are given as [23]:

$$
\begin{gathered}
\rho_{n f}=\rho_{f}(1-\phi)+\rho_{x} \phi \\
\mu_{n f}=\frac{\mu_{f}}{(1-\phi)^{2.5}} \\
v_{n f}=\frac{v_{f}}{\rho_{n f}}
\end{gathered}
$$

Here, $\phi$ is the solid volume fraction. Considering $\frac{\partial u(r, \theta)}{\partial \theta}$ for the purely radial flow, we can define the velocity parameter as:

$$
f(\theta)=r u(r)
$$

Introducing $\eta=\frac{\theta}{\alpha}$ as the dimensionless degree, the dimensionless form of the velocity parameter can be obtained by dividing that to its maximum value as:

$$
f(\eta)=\frac{f(\eta)}{f_{\max }}
$$

Substituting Eq.(7) into (2) and (3) and eliminating $P$, one can obtain the ordinary differential equation for the normalized function profile as [8]:

$$
\begin{aligned}
& f^{\prime \prime \prime}(\eta)+2 \alpha \operatorname{Re} A^{*}(1-\phi)^{2.5} f(\eta) f^{\prime}(\eta)+ \\
& \left.(4-(1-\phi))^{1.25} H a\right) \alpha^{2} f^{\prime}(\eta)=0
\end{aligned}
$$

where $A^{*}$ is a parameter, Reynolds number $R e$ and Hartmann number $\mathrm{Ha}$ based on the electromagnetic parameter are introduced as follows:

$$
\begin{gathered}
A *=(1-\phi)+\frac{\rho_{s}}{\rho_{f}} \phi \\
R e=\frac{f_{\max } \alpha}{v_{f}}=\frac{U_{\max } r \alpha}{v_{f}} \\
H a=\sqrt{\frac{\sigma B_{0}^{2}}{\rho_{f} v_{f}}}
\end{gathered}
$$

with the following reduced form of boundary conditions:

$$
f(0)=1, \quad f^{\prime}(0)=0, \quad f(1)=0
$$

Physically, these boundary conditions mean that maximum values of velocity are observed at center line $\eta=0$ as shown in Figure 1, and we consider fully develop velocity profile. Thus, rate of velocity is zero at $\eta=0$. Also, in fluid dynamics, the no-slip condition for fluid states that at a solid boundary, the fluid will have zero velocity relative to the boundary. The fluid velocity at all fluidsolid boundaries is equal to that of the solid boundary, so we can see that value of velocity is zero at $\eta=1$.

\section{The analytical methods and applications}

\subsection{Homotopy perturbation method (HPM)}

To illustrate basic concepts of the HPM, consider the following differential equation:

$$
L u+N u=g(t)
$$

where $L$ is a linear operator, a nonlinear operator and $g(t)$ is an inhomogeneous term. So, homotopy perturbation structure is shown as in the following equation:

$$
H(v, p)=L(v)-L\left(u_{0}\right)+p L\left(u_{0}\right)+p[N(v)]=0
$$

where $p$ is an embedding parameter and $u_{0}$ is the first approximation that satisfies the boundary condition. The process of changes in $p$ from zero to unity is that of $v(r, p)$ changing from $u_{0}$ to $u(r)$. We consider $v$ as following:

$$
v=v_{0}+p v_{1}+p^{2} v_{2}+p^{3} v_{3}+p^{4} v_{4}+\ldots
$$

And the best approximation for solution is:

$$
u=\lim _{p \rightarrow 1} v=v_{0}+v_{1}+v_{2}+v_{3}+v_{4}+\ldots
$$

By applying HPM into Eq. (9) and substituting (see Eq. (16)) into it and rearranging based on powers of $p-$ terms, it can be obtained,

$$
\begin{aligned}
& p^{0}: \frac{d^{3}}{d \eta^{3}} f_{0}(\eta) \\
& p^{1}: 2 \alpha \operatorname{Re} A^{*}(1-\phi)^{2.5} f_{0}(\eta)\left(\frac{d}{d \eta} f_{0}(\eta)\right) \\
& -\alpha^{2}(1-\phi)^{1.25} \mathrm{Ha}\left(\frac{d}{d \eta} f_{0}(\eta)\right) \\
& +4 \alpha^{2}\left(\frac{d}{d \eta} f_{0}(\eta)\right)+\frac{d^{3}}{d \eta^{3}} f_{1}(\eta) \\
& p^{2}: 4 \alpha^{2}\left(\frac{d}{d \eta} f_{1}(\eta)\right)+2 \alpha \operatorname{Re} A^{*}(1-\phi)^{2.5} f_{1}(\eta)\left(\frac{d}{d \eta} f_{0}(\eta)\right) \\
& +\frac{d^{3}}{d \eta^{3}} f_{2}(\eta)+2 \alpha \operatorname{Re} A^{*}(1-\phi)^{2.5} f_{0}(\eta)\left(\frac{d}{d \eta} f_{1}(\eta)\right) \\
& -\alpha^{2}(1-\phi)^{1.25} H a\left(\frac{d}{d \eta} f_{1}(\eta)\right)
\end{aligned}
$$

and etc. 


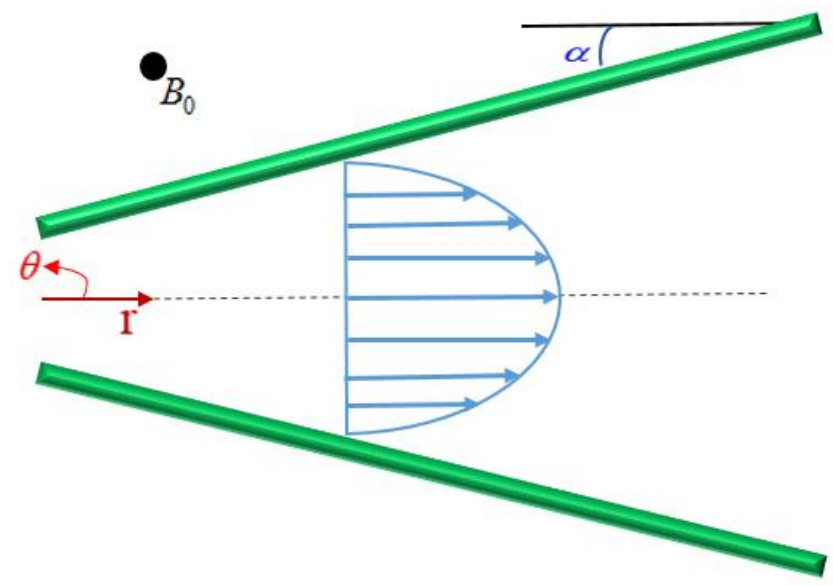

Figure 1. The schematic diagram of the physical model.

Therefore,

$$
\begin{aligned}
& f_{0}(\eta)=-\eta^{2}+1 \\
& f_{1}(\eta)=-2 \alpha\left(\frac { 1 } { 1 2 0 } \left(2 A^{*} R e \phi^{2} \sqrt{1-\phi}-4 A^{*} R e \sqrt{1-\phi}\right.\right. \\
& +2 A \sqrt{1-\phi} R e) \eta^{6}+\frac{1}{24}\left(2 A^{*} R e \phi^{2} \sqrt{1-\phi}\right. \\
& +4 A^{*} \operatorname{Re} \phi \sqrt{1-\phi}-H a(1-\phi)^{\frac{1}{4}} \alpha \phi-2 A^{*} \sqrt{1-\phi} R e \\
& \left.H a(1-\phi)^{\frac{1}{4}} \alpha-4 \alpha\right) \eta^{4} \\
& -\frac{1}{60} \frac{1}{-1+\phi}\left(( 1 - \phi ) ^ { \frac { 1 } { 4 } } \alpha \left(-24 A^{*}(1-\phi)^{\frac{1}{4}} \operatorname{Re} \phi^{2}\right.\right. \\
& +8 A^{*}(1-\phi)^{\frac{1}{4}} \operatorname{Re} \phi^{3}+24 A^{*}(1-\phi)^{\frac{1}{4}} \operatorname{Re} \phi \\
& -8 A^{*}(1-\phi)^{\frac{1}{4}} \operatorname{Re}+5 H a \alpha \phi^{2} \alpha-10 H a \phi \alpha+5 H a \alpha \\
& \left.\left.-20(1-\phi)^{\frac{3}{4}} \alpha\right) \eta^{2}\right)
\end{aligned}
$$

and etc.

The solution of this equation when $p \rightarrow 1$ would be as follows:

$$
f(\eta)=f_{0}(\eta)+f_{1}(\eta)+f_{2}(\eta)+f_{3}(\eta)+f_{4}(\eta)+\ldots
$$

For example, by substituting $A^{*}=1.1, \mathrm{Ha}=500, \mathrm{Re}=$ 50, $\alpha=5^{\circ}, \theta=0.1$ into Eq. (9) following solution would be obtained by HPM,

$$
\begin{aligned}
f= & -1.233827541 \eta^{2}+1-0.2220652426 \eta^{6} \\
& +0.4123741072 \eta^{4}-0.01007475689 \eta^{10} \\
& +0.05359343297 \eta^{8}
\end{aligned}
$$

\subsection{Akbari-Ganji's Method (AGM)}

Boundary conditions and initial conditions are required for analytical methods of each linear and nonlinear differential equation according to the physic of the problem. Therefore, we can solve every differential equation with any degrees. In order to comprehend the given method in this paper, two differential equations governing on engineering processes will be solved in this new manner.

In accordance with the boundary conditions, the general manner of a differential equation is as follows: The nonlinear differential equation of $p$ which is a function of $u$, the parameter $u$ which is a function of $x$ and their derivatives are considered as follows:

$$
p_{k}: f\left(u, u^{\prime}, u^{\prime \prime}, \ldots, u^{(m)}\right)=0 ; \quad u=u(x)
$$

Boundary conditions:

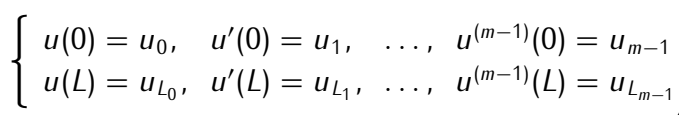

To solve the first differential equation with respect to the boundary conditions in $x=L$ in Eq. (23), the series of letters in the $n$th order with constant coefficients which is the answer of the first differential equation is considered as follows:

$$
u(x)=\sum_{i=0}^{n} \alpha_{i} x^{i}=a_{0}+a_{1} x^{1}+a_{2} x^{2}+\ldots+a_{n} x^{n}
$$


The more precise answer of Eq. (22), the more choice of series sentences from Eq. (24). In applied problems, approximately five or six sentences from the series are enough to solve nonlinear differential equations. In the answer of differential Eq. (24) regarding the series from degree $(n)$, there are $(n+1)$ unknown coefficients that need $(n+1)$ equations to be specified. The boundary conditions of Eq. (23) are used to solve a set of equations which is consisted of $(n+1)$ ones. The boundary conditions are applied on the functions such as follows:

(a) The application of the boundary conditions for the answer of differential Eq. (24) is in the form of: If $x=0$ :

$$
\left\{\begin{array}{lll}
u(0)= & a_{0}=u_{0} \\
u^{\prime}(0)= & a_{1} & u_{1} \\
u^{\prime \prime}(0)= & a_{2} & u_{2} \\
\vdots & \vdots & \vdots
\end{array}\right.
$$

And when $x=L$

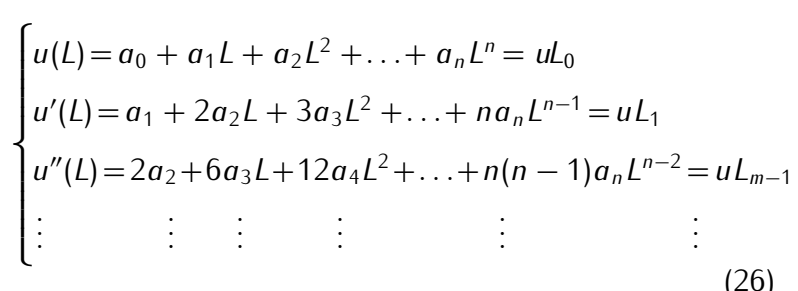

(b) After substituting Eq. (26) into Eq. (22), the application of the boundary conditions on differential Eq. (22) is done according to the following procedure:

$$
\begin{array}{cccccc}
p_{0}: f(u(0), & u^{\prime}(0), & u^{\prime \prime}(0), & \ldots, & \left.u^{(m)}\right) \\
p_{1}: f(u(L), & u^{\prime}(L), & u^{\prime \prime}(L), & \ldots, & \left.u^{(m)}(L)\right) \\
\vdots & & \vdots & \vdots &
\end{array}
$$

With regard to the choice of $n ;(n<m)$ sentences from Eq. (24) and in order to make a set of equations which is consisted of $(n+1)$ equations and $(n+1)$ unknowns, we confront with a number of additional unknowns which are indeed the same coefficients of Eq. (24). Therefore, to remove this problem, we should derive $m$ times from Eq. (22) according to the additional unknowns in the afore-mentioned set differential equations and then this is the time to apply the boundary conditions of Eq. (11) on them.

$$
\begin{array}{lllll}
p_{k}^{\prime}: f\left(u^{\prime},\right. & u^{\prime \prime}, & u^{\prime \prime \prime}, & \ldots, & \left.u^{(m+1)}\right) \\
p_{k}^{\prime \prime}: f\left(u^{\prime \prime},\right. & u^{\prime \prime \prime}, & u^{(I V)}, & \ldots, & \left.u^{(m+2)}\right)
\end{array}
$$

(c) Application of the boundary conditions on the derivatives of the differential equation $p_{k}$ in Eq. (28) is done in the form of:

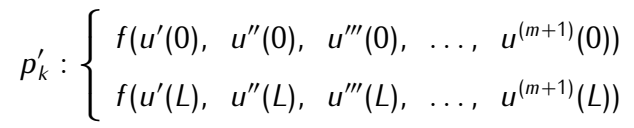

$$
\begin{aligned}
& p_{k}^{\prime \prime}:\left\{\begin{array}{llll}
f\left(u^{\prime \prime}(0),\right. & u^{\prime \prime \prime}(0), & \ldots, & \left.u^{(m+2)}(0)\right) \\
f\left(u^{\prime \prime}(L),\right. & u^{\prime \prime \prime}(L), & \ldots, & \left.u^{(m+2)}(L)\right)
\end{array}\right.
\end{aligned}
$$

$(n+1)$ equations can be made from Eq. (25) to Eq. (29) so that $(n+1)$ unknown coefficients of Eq. (24) take for example $a_{0}, a_{1}, a_{2}, \ldots, a_{n}$ will be computed. The answer of the nonlinear differential Eq. (22) will be gained by determining coefficients of Eq. (24).

Note that according to Eq. (9) we consider $g(\eta)$ as follows:

$$
\begin{aligned}
& g(\eta): f^{\prime \prime \prime}(\eta)+2 \alpha \operatorname{Re} A^{*}(1-\phi)^{2.5} f(\eta) f^{\prime}(\eta) \\
& +\left(4-1(1-\phi)^{2.5} H a\right) \alpha^{2} f^{\prime}(\eta)=0
\end{aligned}
$$

By considering variables $k_{1}$ and $k_{2}$ as follows:

$$
k_{1}=2 \alpha \operatorname{Re} A^{*}(1-\phi)^{2.5}, k_{2}=\left(4-(1-\phi)^{2.5} H a\right) \alpha^{2}
$$

Eq. (31) will convert to the following form:

$$
f(\eta): \frac{d^{3}}{d \eta^{3}} f(\eta)+k_{1} f \frac{d}{d \eta} f(\eta)+k_{2} \frac{d}{d \eta} f(\eta)=0
$$

In AGM, the answer of the differential equations is considered as a finite series of polynomials with constant coefficients as follows:

$f=\sum_{k=0}^{6} a_{k} \eta^{k}=a_{0}+a_{1} \eta+a_{2} \eta^{2}+a_{3} \eta^{3}+a_{4} \eta^{4}+a_{5} \eta^{5}+a_{6} \eta^{6}$

It is notable that in the above equations, the constant coefficients of $a_{0}$ to $a_{6}$, are obtained by applying the introduced boundary conditions.

\section{Applying boundary conditions}

In AGM, the boundary conditions are applied in order to compute constant coefficients of Eq. (34) in two ways as follows:

(a) Applying the boundary conditions on Eq. (34) are expressed as follows:

$$
f=f(B C)
$$


It is notable that $B C$ is the abbreviation of boundary conditions.

According to the above explanations, applying the boundary conditions on Eq. (34) is done in the following form:

$$
\begin{gathered}
f(0)=1 \rightarrow a_{0}=1 \\
f(1)=0 \rightarrow a_{6}+a_{5}+a_{4}+a_{3}+a_{2}+a_{1}+a_{0}=0 \\
f^{\prime}(0)=0 \rightarrow a_{1}=0
\end{gathered}
$$

(b) Applying the boundary conditions on the main differential equation, which in this case study is Eq. (33), and on its derivative is done after substituting Eq. (34) into the main differential equation Eq. (33) as follows:

$$
g(f(\eta)) \rightarrow g(f(B C))=0, \quad g^{\prime}(f(\eta)) \rightarrow g^{\prime}(f(B C))=0
$$

Based on the above formulae, the boundary conditions are applied on the achieved differential equation as follows:

$$
g(f(0)): 6 a_{3}+k_{1} a_{0} a_{1}+k_{2} a_{1}=0
$$

$$
\begin{aligned}
& g(f(1)): 6 a_{3}+24 a_{4}+60 a_{5}+120 a_{6}+ \\
& k_{1}\left(a_{0}+a_{1}+a_{2}+a_{3}+a_{4}+a_{5}+a_{6}\right) . \\
& \cdot\left(a_{1}+2 a_{2}+3 a_{3}+4 a_{4}+5 a_{5}+6 a_{6}\right) \\
& +k_{2}\left(a_{1}+2 a_{2}+3 a_{3}+4 a_{4}+5 a_{5}+6 a_{6}\right)=0
\end{aligned}
$$

Then, the boundary conditions are applied on the derivative of the acquired differential equation in the following form:

$$
\begin{aligned}
& g^{\prime}(f(0)): 24 a_{4}+k_{1} a_{1}^{2}+2 k_{1} a_{0} a_{2}+2 k_{2} a_{2}=0 \\
& g^{\prime}(f(1)): 24 a_{4}+120 a_{5}+36 a_{6} \\
& +k_{1}\left(a_{1}+2 a_{2}+3 a_{3}+4 a_{4}+5 a_{5}+6 a_{6}\right)^{2} \\
& +k_{1}\left(a_{0}+a_{1}+a_{2}+a_{3}+a_{4}+a_{5}+a_{6}\right) . \\
& \cdot\left(2 a_{2}+6 a_{3}+12 a_{4}+20 a_{5}+30 a_{6}\right) \\
& +k_{2}\left(2 a_{2}+6 a_{3}+12 a_{4}+20 a_{5}+30 a_{6}\right)=0
\end{aligned}
$$

By solving a set of algebraic equations which is consisted of seven equations with seven unknowns from Eq. (36-43), the constant coefficients of Eq. (34) can easily be gained.
To simplify, the following new variables are considered in the form of:

$$
\begin{aligned}
& \psi=518400 k_{1}+155520 k_{1}^{2}-k_{2}^{5}-7632 k_{1}^{2} k_{2}-2880 k_{1}^{3} \\
& +233280 k_{1} k_{2}-2160 k_{1} k_{2}^{2}+60 k_{2}^{4}-2 k_{2}^{4} k_{1} \\
& -31104000-k_{1}^{2} k_{2}^{3}+518400 k_{2}-48 k_{1}^{2} k_{2}^{2}+12 k_{2}^{3} k_{1} \\
& +\left(-3.224862720 \cdot 10^{13} k_{1}-3.224862720 \cdot 10^{13} k_{2}\right. \\
& -1.397440512 \cdot 10^{13} k_{1} k_{2}+2.687385600 \cdot 10^{11} k_{2}^{2} \\
& +1.845338112 \cdot 10^{12} k_{1}^{2} k_{2}-1.155575808 \cdot 10^{13} k_{1}^{2} \\
& +3.881779200 \cdot 10^{10} k_{2}^{3} k_{1}-3.981312000 \cdot 10^{8} k_{2}^{4} k_{1} \\
& +4.367001600 \cdot 10^{10} k_{1}^{2} k_{2}^{2}-3.565762560 \cdot 10^{9} k_{1}^{3} k_{2}^{2} \\
& -3.670272000 \cdot 10^{9} k_{1}^{2} k_{2}^{3}-6.136197120 \cdot 10^{10} k_{1}^{3} k_{2} \\
& -1.150848000 \cdot 10^{7} k_{2}^{4} k_{1}^{2}-2.488320000 \cdot 10^{7} k_{2}^{5} k_{1} \\
& +1.866240000 \cdot 10^{6} k_{2}^{5} k_{1}^{2}+7.948800000 \cdot 10^{5} k_{2}^{6} k_{1} \\
& -3.732480000 \cdot 10^{9} k_{2}^{4}+1.244160000 \cdot 10^{8} k_{2}^{5} \\
& +1.021206528 \cdot 10^{12} k_{1}^{3}-1.036800000 \cdot 10^{6} k_{2}^{6} \\
& +1.700352000 \cdot 10^{6} k_{2}^{4} k_{1}^{3}-27792 k_{2}^{6} k_{1}^{2}-5760 k_{2}^{7} k_{1} \\
& -72 k_{2}^{7} k_{1}^{2}-264 k_{2}^{8} k_{1}+2.53808640010^{8} k_{1}{ }^{4} k_{2} \\
& +6.801408000 \cdot 10^{7} k_{1}^{3} k_{2}^{3}-29644 k_{2}^{5} k_{1}^{3}+6 k_{2}^{8} k_{1}^{2} \\
& +4 k_{2}^{9} k_{1}+6.985958400 \cdot 10^{7} k_{1}^{4} k_{2}^{2}+4.216320000 \cdot 10^{5} k_{1}^{4} k_{2}^{3} \\
& +7.464960000 \cdot 10^{6} k_{1}^{5} k_{2}-2.073600000 \cdot 10^{5} k_{1}^{5} k_{2}^{2} \\
& -5760 k_{1}^{5} k_{2}^{3}-16992 k_{1}^{4} k_{2}^{4}+96 k_{1}^{4} k_{2}^{5}+168 k_{1}^{3} k_{2}^{6} \\
& +k_{1}^{4} k_{2}^{6}+4 k_{1}^{3} k_{2}^{7}+3600 k_{2}^{8}-120 k_{2}^{9}+k_{2}^{10} \\
& -2.956124160 \cdot 10^{10} k_{1}^{4}+2.687385600 \cdot 10^{8} k_{1}^{5} \\
& \left.+1.791590400 \cdot 10^{10} k_{1} k_{2}^{2}+9.674588160 \cdot 10^{4}\right)^{0.5} \\
& \Delta_{1}=k_{1}^{2}-120 k_{1}-120 k_{2}+k_{2}^{2}+2 k_{1} k_{2}+3600 \\
& \Delta_{2}=k_{1} k_{2}+36 k_{1}+k_{2}^{2}-720
\end{aligned}
$$

With regards to the above variables, the constant coefficients of Eq. (34) are achieved in the forms of:

$$
\begin{aligned}
& a_{0}=1 \\
& a_{1}=0 \\
& a_{2}=\frac{12}{\Delta_{2}}\left\{\frac{\psi}{k_{1} \Delta_{2}}(0.1041+0.00173)-5 k_{2}-60\right\}, \\
& a_{3}=0 \\
& a_{4}=\frac{-1}{k_{1} \Delta_{1} \Delta_{2}}\left\{5 k_{1}^{2} \Delta_{1}-0.00173 \psi k_{1} k_{2}-0.1042 \psi k_{1}\right. \\
& \left.+5 \Delta_{1} k_{1} k_{2}^{2}-0.001736 \psi k_{2}^{2}-0.10416 \psi k_{2}\right\} \\
& a_{5}=\frac{2}{k_{1} \Delta_{1} \Delta_{2}}\left\{2 k_{2}{ }^{2} k_{1} \Delta_{1}-0.00173 \psi k_{2}{ }^{2}\right. \\
& -0.001736 \psi k_{1} k_{2}+2 \Delta_{1} k_{2} k_{1}^{2}-0.0834 \psi k_{1} \\
& \left.-0.0416 \psi k_{2}+12 \Delta_{1} k_{1}^{2}+1.25 \psi\right\} \\
& a_{6}=\frac{0.001736 \psi}{k_{1} \Delta_{1}}
\end{aligned}
$$


Table 1. The obtained values in accordance with the numerical solution.

\begin{tabular}{lllllll}
\hline$\eta$ & 0 & 0.2 & 0.4 & 0.6 & 0.8 & 1 \\
\hline$f(\eta)$ & 1.000 & 0.949064 & 0.805313 & 0.589751 & 0.321003 & 0.000 \\
$f^{\prime}(\eta)$ & 0.000 & -0.501233 & -0.917467 & -1.221339 & -1.464184 & -1.770954 \\
\hline
\end{tabular}

By substituting the constant values of Eq. (45) into Eq. (34), the differential equation is answered in the following form:

$$
\begin{aligned}
& f(\eta)=1+\frac{12}{\Delta_{2}}\left\{\frac{\psi}{k_{1} \Delta_{2}}(0.1041+0.00173)-5 k_{2}-60\right\} \eta^{2}+ \\
& \frac{-1}{k_{1} \Delta_{1} \Delta_{2}}\left\{5 k_{1}{ }^{2} \Delta_{1}-0.00173 \psi k_{1} k_{2}-0.1042 \psi k_{1}+5 \Delta_{1} k_{1} k_{2}^{2}\right. \\
& \left.-0.001736 \psi k_{2}^{2}-0.10416 \psi k_{2}\right\} \eta^{4} \\
& \frac{2}{k_{1} \Delta_{1} \Delta_{2}}\left\{2 k_{2}^{2} k_{1} \Delta_{1}-0.00173 \psi k_{2}^{2}-0.001736 \psi k_{1} k_{2}\right. \\
& +2 \Delta_{1} k_{2} k_{1}^{2}-0.0834 \psi k_{1}-0.0416 \psi k_{2}+12 \Delta_{1} k_{1}^{2} \\
& +1.25 \psi\} \eta^{5}+\left(\frac{0.001736 \psi}{k_{1} \Delta_{1}}\right) \eta^{6}
\end{aligned}
$$

By selecting the following physical quantities:

$$
H a=500, R e=50, \alpha=5^{\circ}, \phi=0.1
$$

According to Eq. (10) $A^{*}$ is defined as follows:

$$
A^{*}=(1-\phi)+\frac{\rho_{s}}{\rho_{f}} \phi
$$

By substituting $\rho_{s}=2 \rho_{f}$ into Eq. (48) the value of $A^{*}$ would be obtained as follows:

$$
A^{*}=1.1
$$

With the physical values of Eq. (47) and Eq. (49) The answer function is obtained as follows:

$f(\eta)=1-1.22477 \eta^{2}+0.4153 \eta^{4}-0.1148 \eta^{5}-0.07572 \eta^{6}$

Solving the differential equation with Numerical Method and comparing the obtained solutions by HPM and AGM with Numerical Method

With regard to the given physical values of Eq. (47) and Eq. (49) and the introduced domain of $\eta \in\{0,1\}$, the numerical solutions of the mentioned problem is obtained as follows (see Table 1-Table 3 and Figure 2-Figure 8). The following charts are the difference between the results on the basis of the yielded solution from Eq. (50) by AGM and the results of Table 1 by Numerical Method.

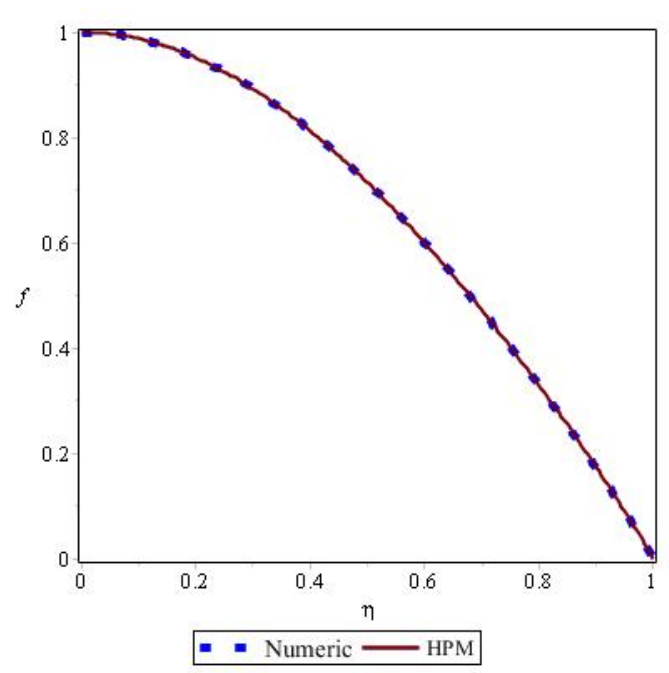

Figure 2. A comparison between the achieved solutions for $f(\eta)$ by HPM and numerical method.

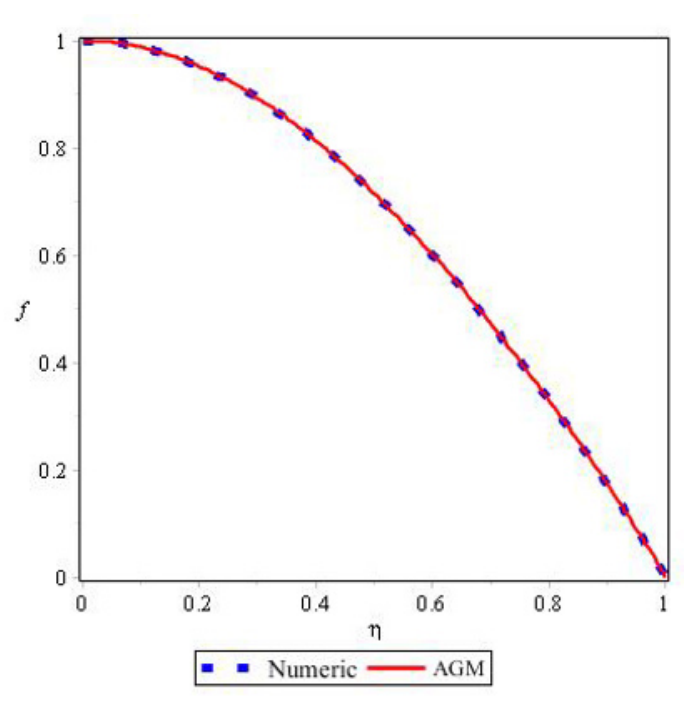

Figure 3. A comparison between the achieved solutions for $f(\eta)$ by AGM and numerical method. 
Table 2. Comparing the obtained values for $f(\eta)$ by HPM and AGM with the numerical solution.

\begin{tabular}{llllll}
\hline$\eta$ & HPM & AGM & Numeric & $\begin{array}{l}\text { Error } \\
\text { percentage of HPM percentage of AGM }\end{array}$ \\
\hline 0 & 1.000 & 1.000 & 1.000 & - & - \\
0.2 & 0.951292 & 0.951632 & 0.949064 & 0.23 & 0.27 \\
0.4 & 0.812268 & 0.813182 & 0.805313 & 0.86 & 0.97 \\
0.6 & 0.599744 & 0.600445 & 0.589751 & 1.69 & 1.81 \\
0.8 & 0.328955 & 0.328783 & 0.321003 & 2.47 & 2.42 \\
1 & 0 & 0.000 & 0.000 & - & - \\
\hline
\end{tabular}

Table 3. Comparing the obtained values for $f(\eta)$ by HPM and AGM with the numerical solution.

\begin{tabular}{llllll}
\hline$\eta$ & HPM & AGM & Numeric & \multicolumn{2}{c}{$\begin{array}{c}\text { Error percentage Error percentage } \\
\text { of HPM }\end{array}$} \\
\hline 0 & & & - & - \\
of AGM
\end{tabular}

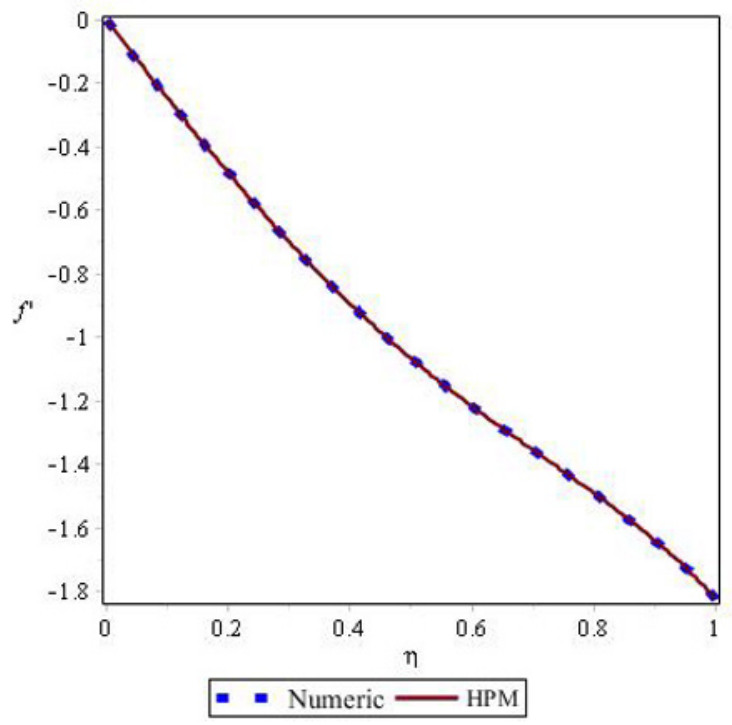

Figure 4. A comparison between the achieved solutions for $f^{\prime}(\eta)$ by HPM and numerical method.

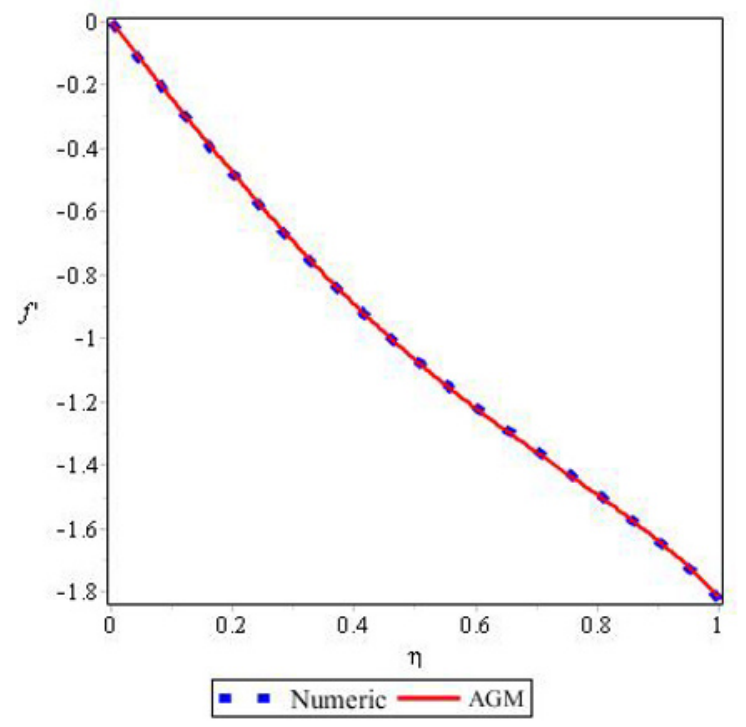

Figure 5. A comparison between the achieved solutions for $f^{\prime}(\eta)$ by AGM and numerical method. 


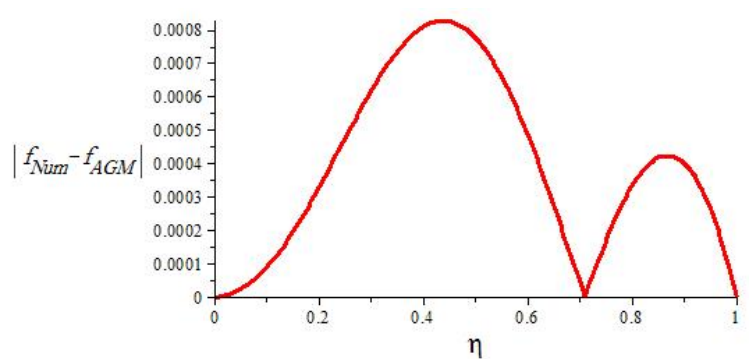

Figure 6. The absolute difference between the obtained solutions by AGM and Numeric Method for $f(\eta)$.

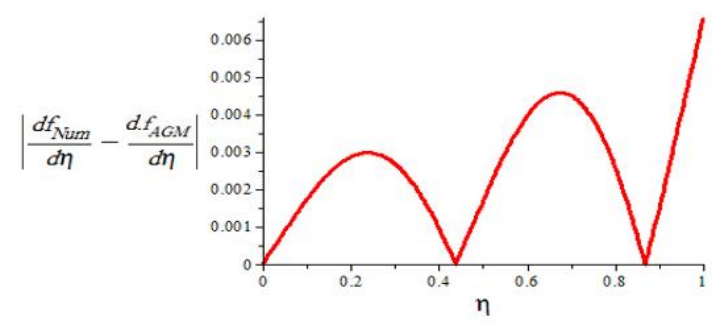

Figure 7. The absolute difference between the obtained solutions by AGM and Numeric Method for $f^{\prime}(\eta)$.

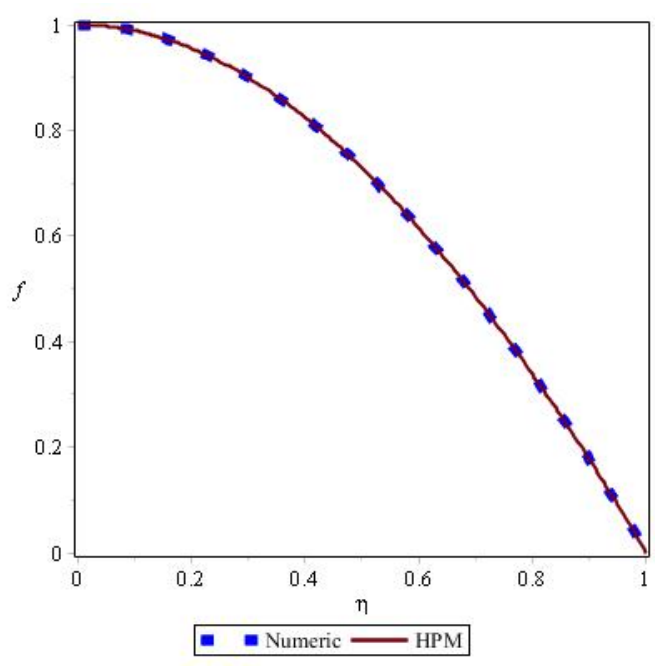

Figure 8. Comparison between the obtained solutions for $f(\eta)$ by HPM and Numerical Method $\left(A^{*}=1, H a=0, R e=50\right.$, $\alpha=1^{\circ}, \phi=0$ ).

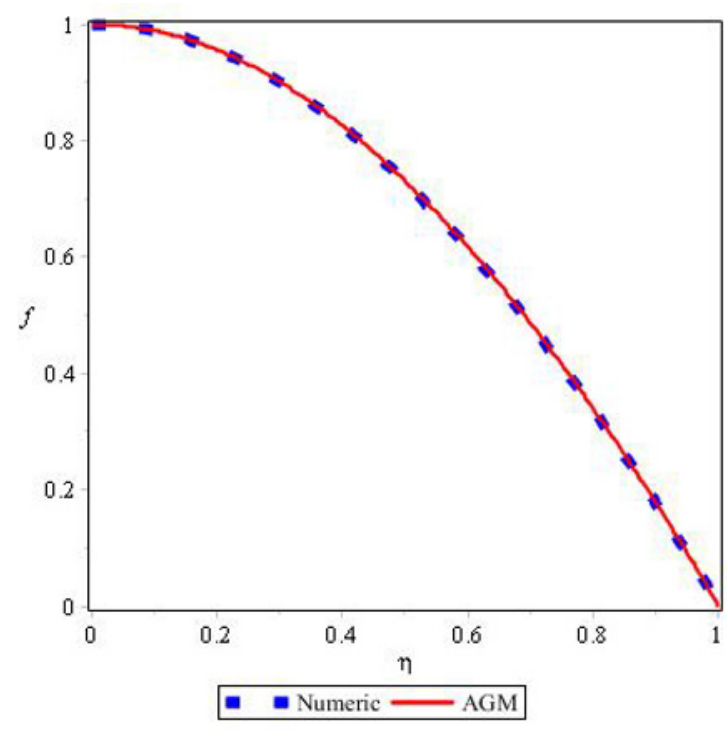

Figure 9. Comparison between the obtained solutions for $f(\eta)$ by AGM and Numerical Method.

\section{Results and discussion}

Graphs for different values of Reynolds number and $\alpha$ are presented to show the accuracy of HPM and AGM. As it is completely clear both methods are accurate and applicable. Also, it is worthwhile to mention that both methods can be more accurate.

Now we present graphs to investigate the effect of different parameters. Figure 18 shows the effect of Reynolds number at constant $\alpha$ and Hartmann number $\left(\alpha=1^{\circ}\right.$, $H a=0)$. Figure 19 and Fig 20 show the effect of Hartmann number at constant $\alpha$ and Reynolds number $(\alpha=$ $\left.1^{\circ}, 5^{\circ}, \operatorname{Re}=200\right)$. Figure 19 shows that at small amounts of $\alpha, f(\eta)$ is almost independent of Hartmann number, but Figure 20 shows that at greater amounts of $\alpha, f(\eta)$ is dependent on Hartmann number. Figure 21. shows the effect of $\alpha$ for constant Reynolds number and Hartmann number $(\operatorname{Re}=200, H a=0)$. Figure 22 and Figure 23 show the effect of $\phi$, for constant $\alpha$, Reynolds number and Hartmann number $\left(\alpha=5^{\circ}, \operatorname{Re}=200 \mathrm{Ha}=0\right)$. Note that $A^{*}$ is dependent on $\phi$, at first we chose $\rho_{s}=2 \rho_{f}$ (Figure 22) and then we chose $\rho_{s}=8933$ (density of $\mathrm{Cu}$.) and $\rho_{s}=997.1$ (density of water). Therefore, Figure 23 shows $f(\eta)$ for $\mathrm{Cu}$-water. 


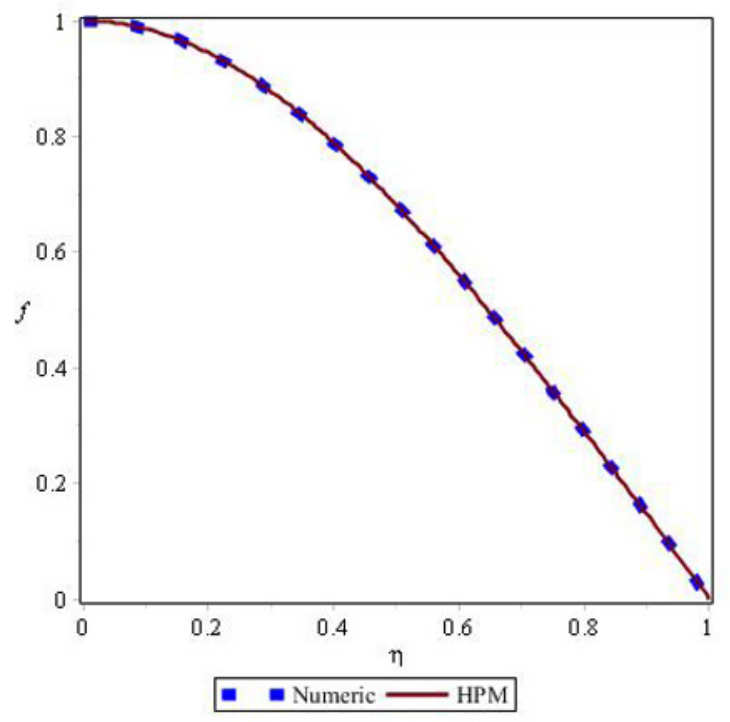

Figure 10. Comparison between the obtained solutions for $f(\eta)$ by HPM and Numerical Method ( $A^{*}=1, H a=0, R e=150$, $\left.\alpha=1^{\circ}, \phi=0\right)$.

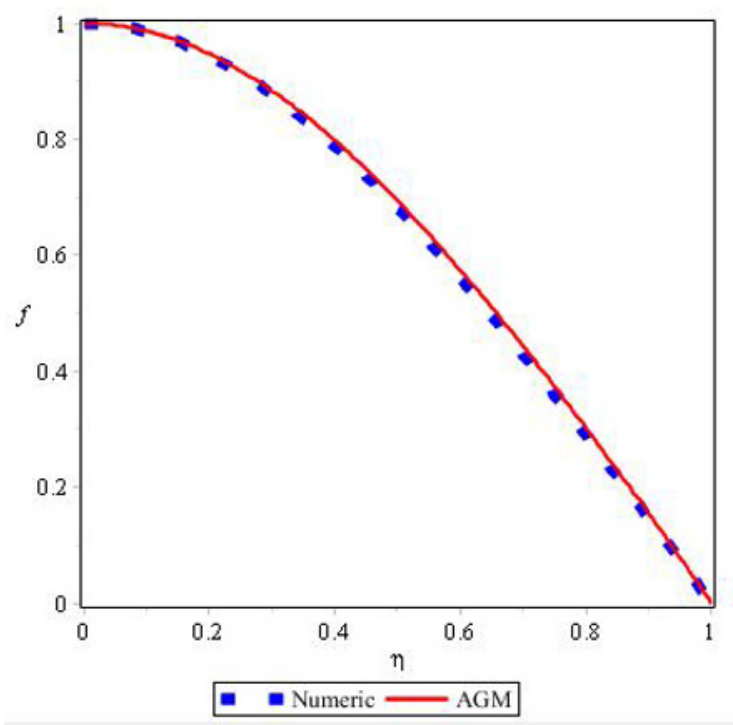

Figure 11. Comparison between the obtained solutions for $f(\eta)$ by AGM and Numerical Method $\left(A^{*}=1, H a=0, R e=150\right.$, $\left.\alpha=1^{\circ}, \phi=0\right)$.

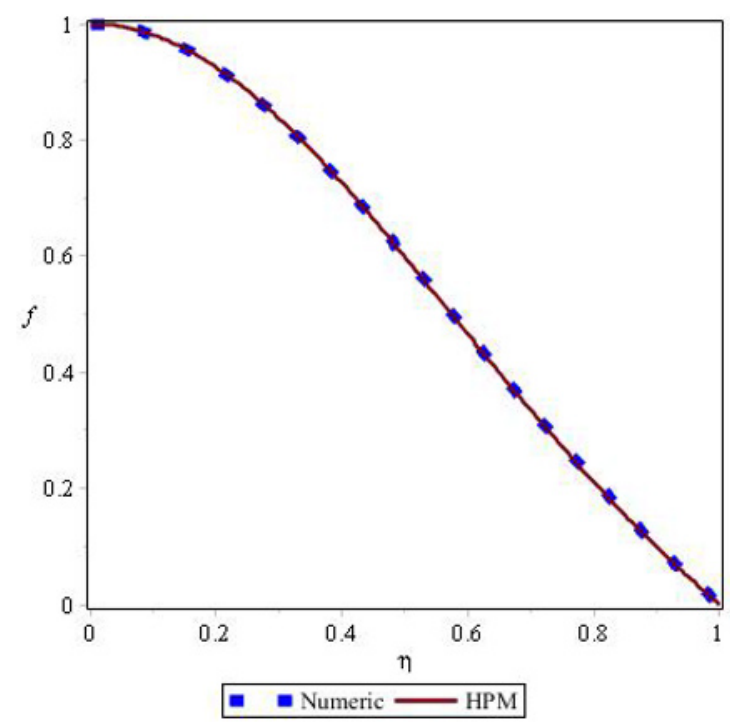

Figure 12. Comparison between the obtained solutions for $f(\eta)$ by HPM and Numerical Method $\left(A^{*}=1, H a=0, R e=150\right.$, $\left.\alpha=1^{\circ}, \phi=0\right)$.

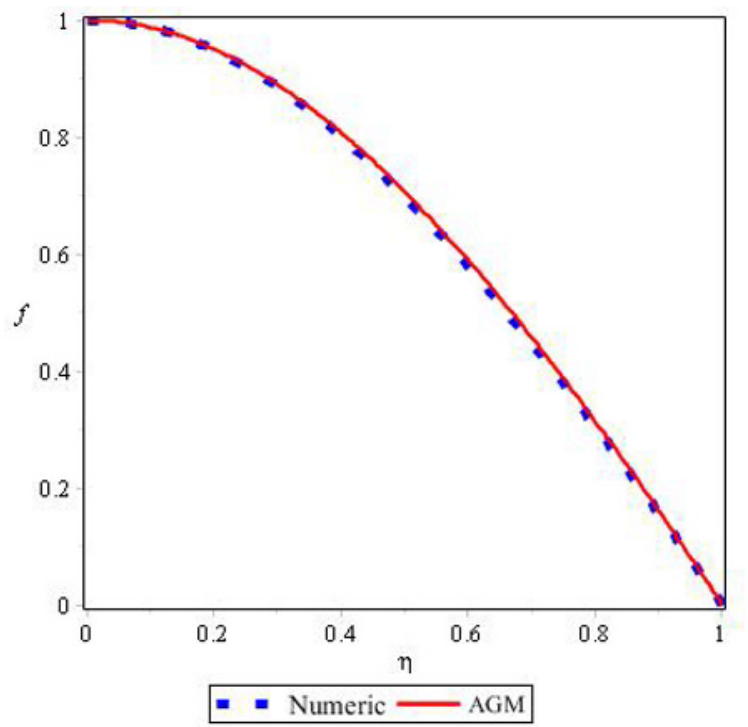

Figure 13. Comparison between the obtained solutions for $f(\eta)$ by AGM and Numerical Method $\left(A^{*}=1, H a=0, R e=150\right.$, $\left.\alpha=1^{\circ}, \phi=0\right)$. 


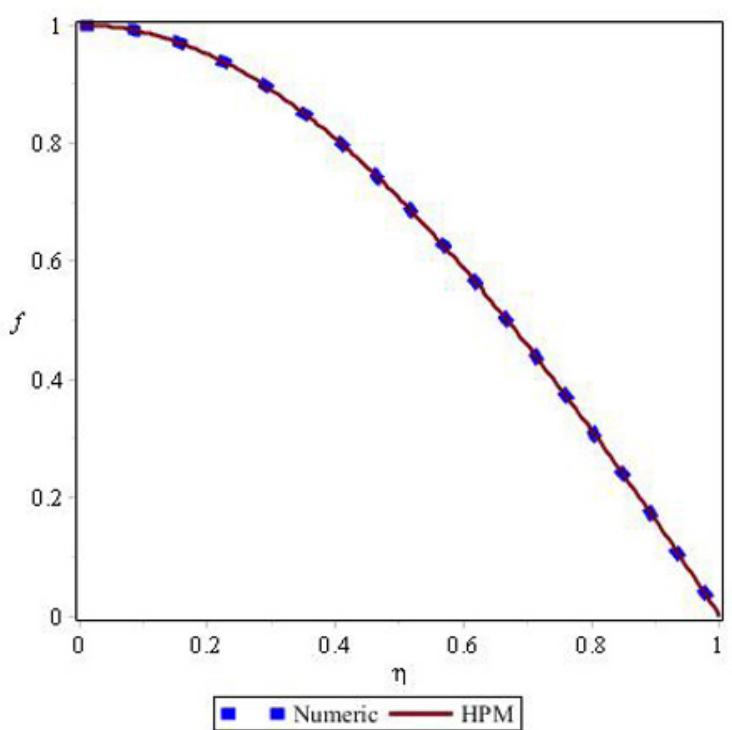

Figure 14. Comparison between the obtained solutions for $f(\eta)$ by HPM and Numerical Method $\left(A^{*}=1, \mathrm{Ha}=0, \operatorname{Re}=50\right.$ $\alpha=2^{\circ}, \phi=0$ ).

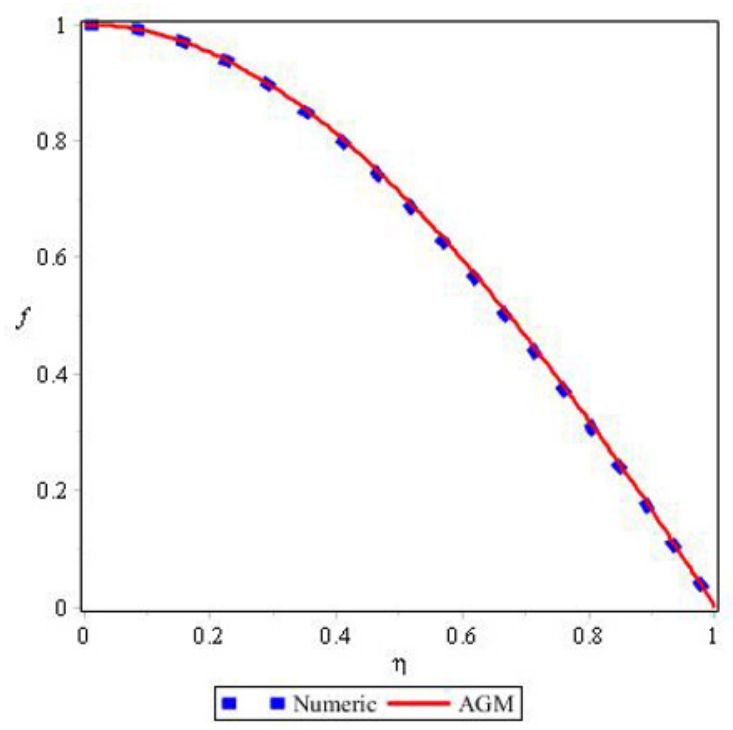

Figure 15. Comparison between the obtained solutions for $f(\eta)$ by AGM and Numerical Method $\left(A^{*}=1, H a=0, R e=50\right.$, $\alpha=2^{\circ}, \phi=0$ ).

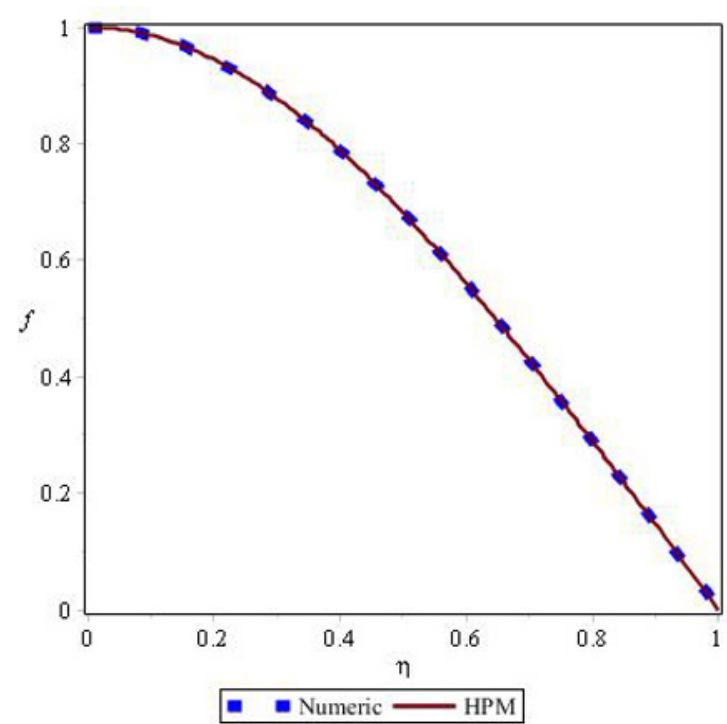

Figure 16. Comparison between the obtained solutions for $f(\eta)$ by HPM and Numerical Method ( $A^{*}=1, H a=0, R e=50$, $\left.\alpha=3^{\circ}, \phi=0\right)$.

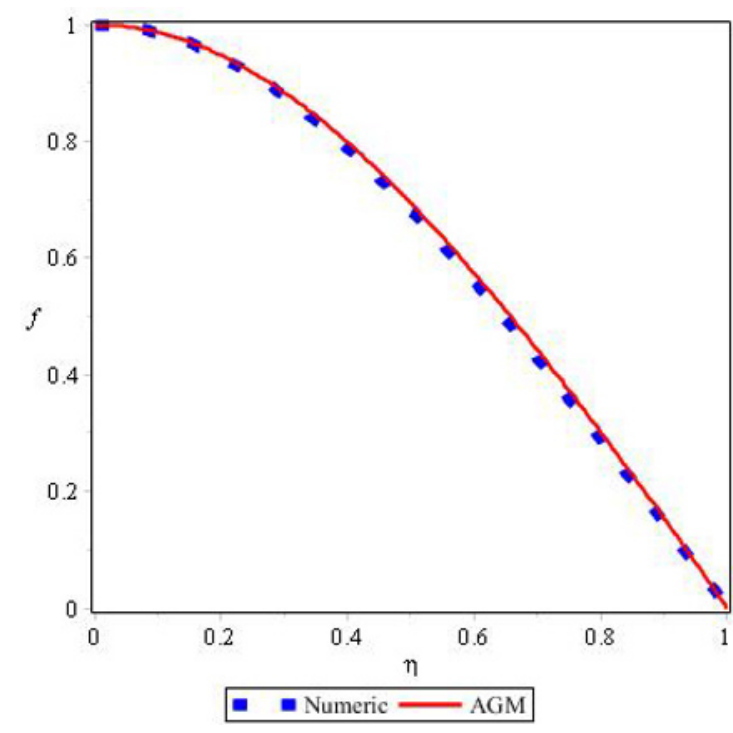

Figure 17. Comparison between the obtained solutions for $f(\eta)$ by HPM and Numerical Method $\left(A^{*}=1, H a=0, R e=50\right.$, $\alpha=3^{\circ}, \phi=0$ ). 


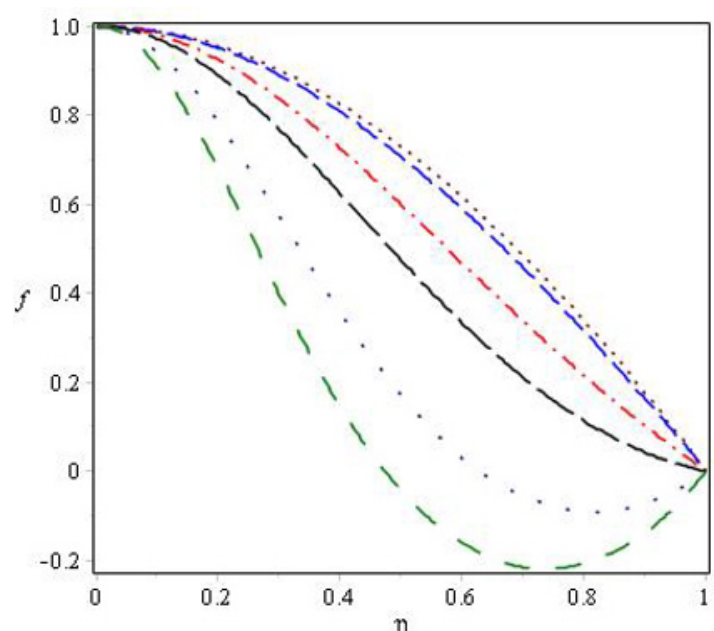
$\cdots \cdot \operatorname{Re}=50--\operatorname{Re}=100-\cdot-\operatorname{Re}=300--\operatorname{Re}=500$
$\cdots \cdot \operatorname{Re}=1000--\operatorname{Re}=1500$

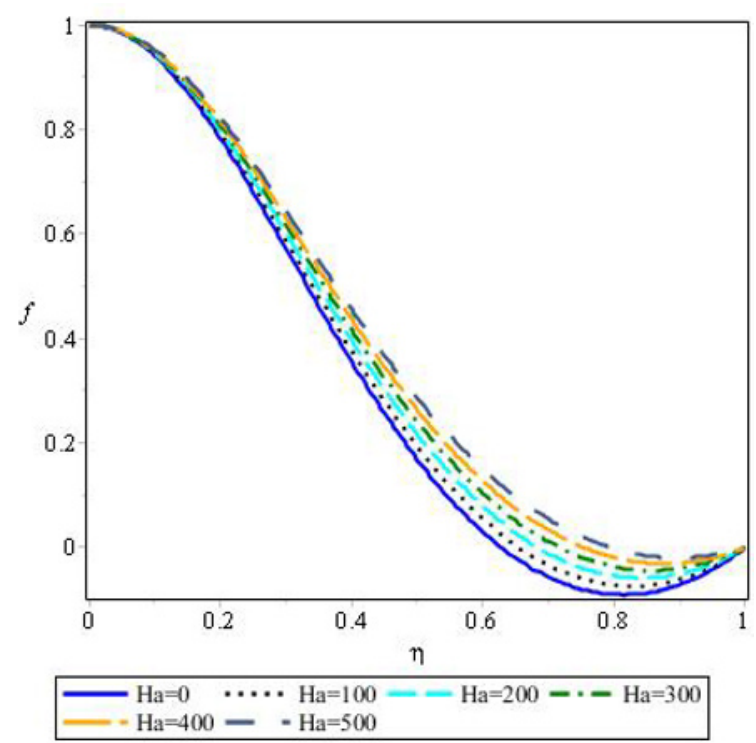

Figure 18. $f(\eta)$ for different values of $\operatorname{Re}\left(A^{*}=1, H a=0, \alpha=1^{\circ}\right.$, $\phi=0$ ).

Figure 20. $f(\eta)$ for different values of $H a\left(A^{*}=1, R e=200, \alpha=5^{\circ}\right.$, $\phi=0$ ).
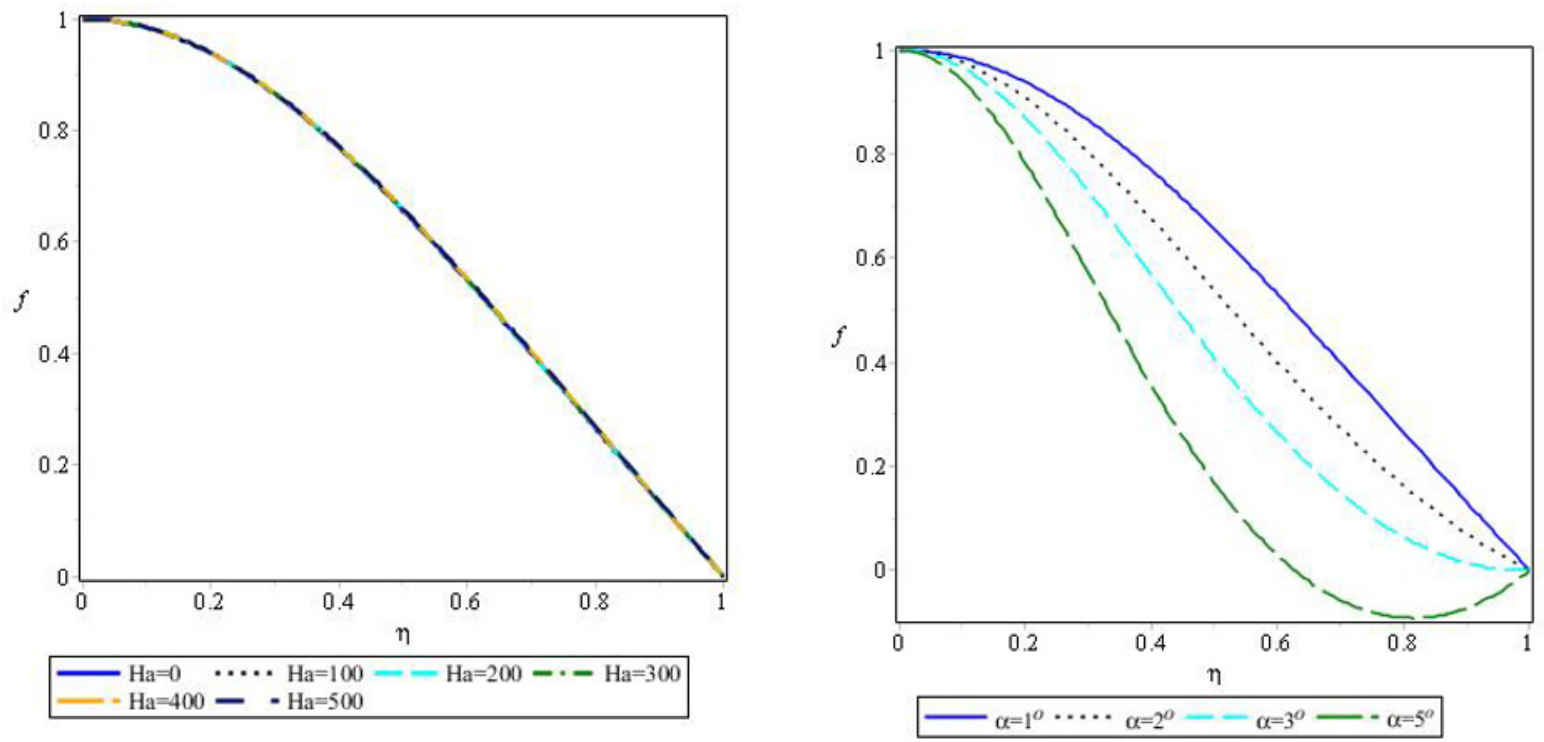

Figure 19. $f(\eta)$ for different values of $H a\left(A^{*}=1, R e=200, \alpha=1^{\circ}\right.$, $\phi=0)$.

Figure 21. $f(\eta)$ for different values of $\alpha\left(A^{*}=1, R e=200, H a=0\right.$, $\phi=0$ ). 


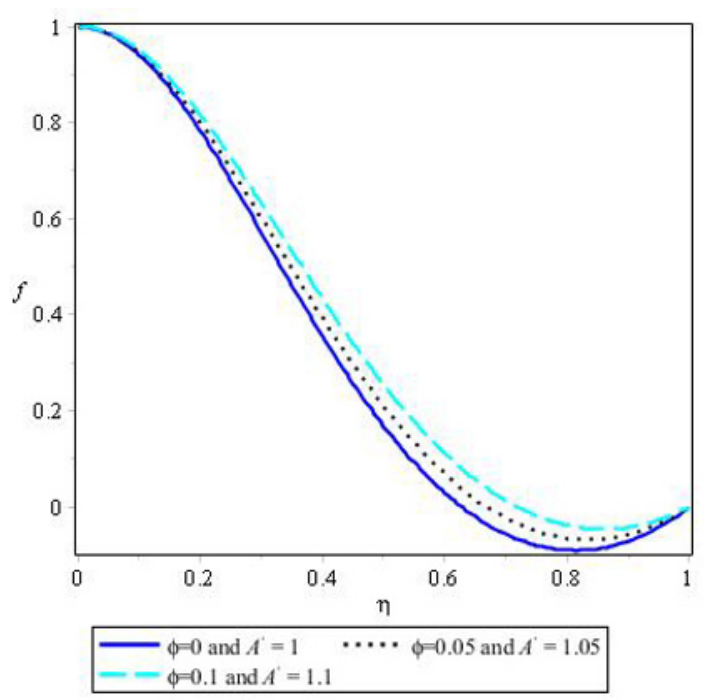

Figure 22. $f(\eta)$ for different values of $\phi$ and $A^{*}(H a=0, R e=200$, $H a=0, \alpha=5^{\circ}$ ).

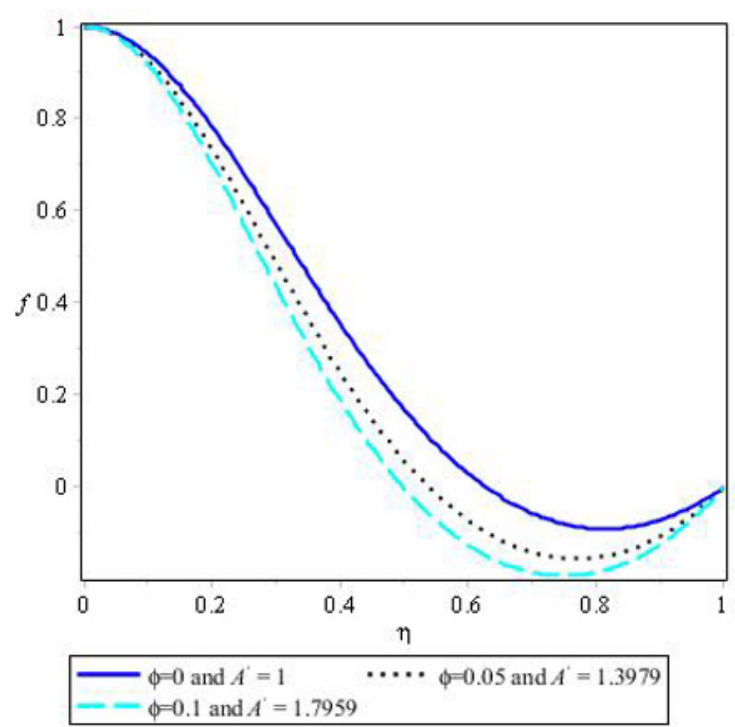

Figure 23. $f(\eta)$ for different values of $\phi$ and $A^{*}(H a=0, R e=200$, $\alpha=5^{\circ}$ ).

\section{Conclusions}

In this case study, two methods have been offered in order to solve a nonlinear differential equation governing on Jeffery-Hamel flow with high magnetic field and nanoparticle, Homotopy Perturbation Method (HPM) and a new method named Akbari-Ganji's Method (AGM). The aforementioned proceeding has been done to indicate the ability of HPM and AGM for solving a broad range of differential equations. We showed that both methods are efficient and accurate. In AGM in contrast to most of the analytical methods which are able to solve differential equations only by introducing dimensionless parameters, a wide variety of complicated differential equations is easily solvable directly which means there is no need to convert variables into new ones. In AGM considering three or four sentences of the series of polynomials which are considered as the answer of differential equations is sufficient to obtain reliable solutions, and always by increasing the number of sentences the solution gets closer to the exact solution while in most of the other methods the error might increase or decrease.

\section{References}

[1] Jeffery G. B., The two-dimensional steady motion of a viscous fluid, Phil. Mag., 6, 1915, 455-465

[2] Hamel G., Spiralformige Bewgungen zaher Flussigkeiten, Jahresber, Deutsch. Math. Verein, 25, 1916, 34-60

[3] Bansal L., Magnetofluiddynamics of Viscous Fluids, Jaipur Publishing House, Jaipur, India, 1994

[4] Cha J. E., Ahn Y. C., and Kim M. H., Flow measurement with an electromagnetic flowmeter in two-phase bubbly and slug flow regimes, Flow Measurement and Instrumentation, 12(5-6), 2002, 329-339

[5] Tendler M., Confinement and related transport in extrap geometry, Nuclear Instruments and Methods in Physics Research, 207(1-2), 1983, 233-240

[6] Makinde O. D. and Motsa S. S., Hydromagnetic stability of plane Poiseuille flow using Chebyshev spectral collocation method, J. Ins. Math. Comput. Sci., 12(2), 2001, 175-183

[7] Makinde O. D., Magneto-hydrodynamic stability of plane-Poiseuille flow using multi-deck asymptotic technique, Math. Comput. Model., 37(3-4), 2003, 251259

[8] Anwari M., Harada N., and Takahashi S., Performance of a magnetohydrodynamic accelerator using air-plasma as working gas, Energy Conversion 
Management, 4, 2005, 2605-2613

[9] Homsy A., Koster S., Eijkel J. C. T., Ven der Berg A., Lucklum F., Verpoorte E., and de Rooij N. F., A high current density DC magnetohydrodynamic (MHD) micropump, Royal Society of Chemistry's Lab on a Chip, 5, 2005, 466-471

[10] Kakac S. and Pramuanjaroenkij A., Review of convective heat transfer enhancement with nanofloids, International Communications in Heat and Mass Transfer, 52(13-14), 2009, 3187-3196

[11] He J. H., Homotopy perturbation technique, Comput. Methods Appl. Mech. Eng., 178, 1999, 257-262

[12] He J. H., Homotopy perturbation method: a new nonlinear analytical technique, Appl. Math. Comput., 135, 2003, 73-79

[13] He J. H., Application of homotopy perturbation method to nonlinear wave equations, Chaos Solitons Fractals 26, 2005, 695-700

[14] He J. H., Homotopy perturbation method for bifurcation of nonlinear problems, Int. J. Nonlinear Sci. Numer. Simul., 6, 2005, 207-208

[15] Roozi A., Alibeiki E., Hosseini S. S., Shafiof S. M., Ebrahimi M., Homotopy perturbation method for special nonlinear partial differential equations, J. King Saud Univ. (Sci.), 23, 2011, 99-103

[16] Ganji D. D., Sadighi A., Application of homotopyperturbation and variational iteration methods to nonlinear heat transfer and porous media equations, J. Comput. Appl. Math., 207, 2007, 24-34

[17] Sadighi A., Ganji D .D., Analytic treatment of linear and nonlinear Schrodinger equations: a study with homotopy-perturbation and Adomian decomposition methods equations, Phys. Lett. A, 372, 2008, 465-469

[18] Sadighi A., Ganji D. D., Exact solutions of Laplace equation by homotopy-perturbation and Adomian decomposition methods, Phys. Lett. A, 367, 2007, 8387

[19] Ziabakhsh Z., Domairry G. , Analytic solution of natural convection flow of a non-Newtonian fluid between two vertical flat plates using homotopy analysis method, Commun. Nonlinear Sci. Numer. Simul., 14, 2009, 1868-1880

[20] Ganji S. S., Ganji D. D., Sfahani M. G., Karimpour S., Application of AFF and HPM to the systems of strongly nonlinear oscillation, Curr. Appl. Phys., 10, 2010, 1317-1325

[21] Khavaji A., Ganji D.D., Roshan N., Moheimani R., Hatami M., A. Hasanpour, Slope variation effect on large deflection of compliant beam using analytical approach, Struct. Eng. Mech., 44(3), 2012, 405-416

[22] Moghimi S. M., Ganji D. D., Bararnia H., Hosseini M., and Jalaal M., Homotopy perturbation method for nonlinear MHD Jeffery-Hamel problem, Computers and Mathematics with Applications, 61(8), 2011, 2213-2216

[23] Aminossadati S. M. and Ghasemi B. Natural convection cooling of a localized heat source at the bottom of a nanofluid-filled enclosure, Eur. J. Mech. B/Fluids, 28, 2009, 630-640 